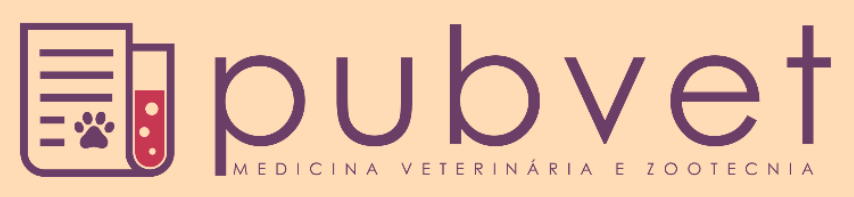

https://doi.org/10.31533/pubvet.v14n2a501.1-7

\title{
Morfologia do fígado e o sistema porta-hepático em Trachemys scripta elegans (Testudinata)
}

\author{
Henrique Fank Paganotto ${ }^{\circ}$, Alan Peres Ferraz de Melo $^{2}{ }^{\circ}$, Luciano de Morais-Pinto ${ }^{30}$, \\ Rosângela Felipe Rodrigues ${ }^{4 * 0}$ \\ 1Médico Veterinário Autônomo. \\ 2Faculdade de Engenharia de Ilha Solteira/UNESP- Departamento de Biologia e Zootecnia, Ilha Solteira - SP. \\ 3 Universidade Federal de Santa Maria, Departamento de Morfologia; Santa Maria - RS, Brasil \\ 4Faculdade de Medicina Veterinária de Araçatuba/UNESP; Departamento de Produção e Saúde Animal. Araçatuba - SP. \\ *Autor para correspondência: Faculdade de Medicina Veterinária. UNESP, Rua Clóvis Pestana, 793; 16050-680, Araçatuba, \\ SP, Brasil.e-mail: rosangela.rodrigues@unesp.br
}

Resumo. Neste estudo foram usados 15 cágados (fêmeas e machos) adultos da espécie Trachemys scripta elegans. Os animais foram conservados em formaldeído (5\%) para análise anatômica e topográfica do fígado e os seus vasos foram injetados com neoprene látex e acetato de vinila para evidenciar o sistema porta-hepático e seus ramos. A veia portahepática drena ( $100 \%$ dos casos) e emite mais dois pontos de penetração na face visceral do fígado, um próximo à vesícula biliar (à direita) e o outro estabelecendo entre ambos a veia porta-hepática transversa (à esquerda). Ela recebe (da esquerda para a direita) as veias gástricas, a veia pancreática duodenal cranial e o tronco das veias jejunais. Este tronco recebe um número variável de ramos das veias jejunais, esplênicas e as mesentéricas cranial e caudal.

Palavras chave: Trachemys scripta elegans, fígado, veia porta-hepática, cágados

\section{Morphology of liver and portal-hepatic system in Trachemys scripta elegans (Testudinata)}

\begin{abstract}
A total of 15 adult female and male red-eared turtles of the species Trachemys scripta elegans (Wied, 1838) were used in this study. The animals were preserved in 5\% formaldehyde for anatomical and topographical analysis of the liver and its vessels were injected with neoprene latex and vinyl acetate to evidence the hepatic portal system and its branches. The hepatic portal vein drains (100\% of cases) and presents two points of penetration into the liver visceral surface, one near the gallbladder (right) and the other establishing between them the transverse hepatic portal vein (left). It receives (from left to right) the gastric and cranial pancreaticoduodenal veins and the union of the jejunal veins. This union receives a variable number of branches from the jejunal, splenic, and cranial and caudal mesenteric veins.
\end{abstract}

Key words: Trachemys scripta elegans, liver, hepatic portal vein, tortoise

\section{Morfología del hígado y del sistema portal-hepático en Trachemys scripta elegans (Testudinata)}

Resumen. En este estudio se utilizaron 15 tortugas de orejas rojas (hembras y machos) adultas de la especie Trachemys scripta elegans (Wied, 1838). Los animales fueron preservados en formaldehído al 5\% para análisis anatómico y topográfico del hígado y sus 
vasos fueron inyectados con látex de neopreno y acetato de vinilo para evidenciar el sistema portal hepático y sus ramas. La vena porta hepática drena (100\% de los casos) y presenta dos puntos de penetración en la superficie visceral del hígado, uno cerca de la vesícula biliar (derecha) y el otro que establece entre ellos la vena porta hepática transversal (izquierda). Recibe (de izquierda a derecha) las venas pancreaticoduodenales gástricas y craneales y la unión de las venas yeyunales. Esta unión recibe un número variable de ramas de las venas mesentéricas yeyunal, esplénica y craneal y caudal.

Palabras clave: Trachemys scripta elegans, hígado, vena porta hepática, tortuga

\section{Introdução}

Os cágados, ou tartarugas de água doce, apresentam hábitos semi-aquáticos e possuem membros torácicos e pélvicos com membranas interdigitais e unhas, que facilitam o deslocamento em água rasa. A espécie Trachemys scripta elegans é popularmente chamada de tartaruga de orelha vermelha e é originada do sul dos Estados Unidos, no Rio Mississippi, leste do Kentucky e norte do Golfo do México (Cubas et al., 2014).

O fígado é peculiar aos vertebrados, e é o maior órgão do corpo. Desempenha diversas funções, como a transformação de proteína em carboidrato ou gordura e a elaboração de grande parte do vitelo do corpo materno para os ovos em desenvolvimento (Hildebrand \& Goslow, 2006). O fígado e o pâncreas são os maiores órgãos da cavidade celomática dos répteis. O fígado é constituído por duas estruturas lobares com coloração variando de castanhos escuros a negro em animais adultos (Zug et al., 2001). O fígado em serpentes apresenta-se em forma alongado no sentido craniocaudal, afinando-se dorsoventral. A veia cava caudal e a veia porta-hepática localizam-se no plano dorsal e a veia hepática no plano ventral. Esses vasos atravessam o parênquima hepático dividindo-o em dois lobos (Isaza et al., 1993).

A veia porta-hepática, presente em todos os vertebrados, não drena apenas o fígado, mas também a região pélvica; nos répteis, a região abdominal também é drenada pela grande veia porta-hepática (Hildebrand \& Goslow, 2006; Pough et al., 2003).

As informações sobre a anatomia e fisiologia dos órgãos digestórios são necessárias, como o estudo de Machado Júnior et al. (2005) em muçuã e Phrynops geoffroanus por Moura et al. (2012). Nas Trachemys scripta elegans o estudo da morfologia do fígado e do sistema porta-hepático é de suma importância no metabolismo orgânico, uma vez que todo alimento que foi absorvido pelo estomago e intestino é metabolizado via hepática.

\section{Material e métodos}

Para a análise anatômica e vascular da veia porta-hepática foram utilizados 15 (quinze) cágados adultos, machos e fêmeas, da espécie Trachemys scripta elegans (Wied, 1838). Os animais foram fornecidos pelo Zoológico de Bauru e o Instituto Brasileiro do Meio Ambiente e dos Recursos Renováveis (IBAMA), de acordo com a licença $n^{\circ}$ 006/02 e processo $n^{\circ}$ 022012001113/2002-81, autorizado. O protocolo de experimentação animal $n^{\circ}$ 048/2009 foi emitido pelo Comitê de Ética em Pesquisa do Centro Universitário Barão de Mauá. Os animais foram submetidos à eutanásia de acordo com o protocolo de Tranquilli et al. (2013), com cloridrato de xilazina (1 mg/kg) e cloridrato de cetamina $(20 \mathrm{mg} / \mathrm{kg})$ por via intramuscular no membro pélvico do antímero esquerdo. Após relaxamento, os animais foram eutanasiados com a aplicação de dose letal $(60 \mathrm{mg} / \mathrm{kg})$ de tiopental sódico a $2,5 \%$ pela veia jugular externa. Em seguida os animais tiveram o plastrão removido com uma serra de aço para a desarticulação da ponte óssea que une o plastrão à carapaça, e com o bisturi removeu-se os tecidos musculares para o acesso à cavidade celomática. Esta manobra permitiu observar a disposição topográfica dos órgãos. Injetou-se em cinco animais substância látex corada de azul e branco, e em outros cinco animais optou-se por injetar acetato de vinila, nas cores azul branca e vermelha. Para as injeções foram utilizadas agulhas $40 \times 12$ sem a parte lancetada, por meio da veia abdominal com aproximadamente $10 \mathrm{ml}$ de cada substância de com colorações diferentes para os preenchimentos do sistema porta-hepático, veias jejunais, baço e também o sistema porta-renal. Os cadáveres injetados com substância látex foram mantidos em formaldeído a 5\% por um período mínimo de três horas, para em seguida realizar uma dissecação minuciosa da veia porta-hepática e suas ramificações, bem como a 
descrição anatômica do fígado. Os animais injetados com acetato de vinila tiveram o conjunto de vísceras digestórias retiradas, submergidas em solução a 5\% de ácido sulfúrico para a corrosão dos tecidos viscerais, por 15 dias. Após este período o material foi lavado em água corrente por 12 horas para retirada do excesso de ácido sulfúrico e resíduos, em seguida estava preparado para análise e foto documentação.

Os resultados encontrados foram registrados na documentação fotográfica e as estruturas obtidas foram nomeadas partir do World Association of Veterinary Anatomists. International Committee on Avian Anatomical Nomenclature (Nomina Anatomica Avium, (Baumel, 1993), pois esta espécie não possui uma nomenclatura anatômica condizente.

\section{Resultados e discussão}

\section{Aspecto morfológico e topográfico do fígado}

A coloração hepática nas Trachemys scripta elegans apresentou-se marrom claro ou marrom avermelhada como evidenciado por (Silva et al., 2011) e Moura et al. (2012), sendo na Phrynops geoffroannus, de coloração marrom pálida mesclada com manchas pretas (Moura et al., 2017). No cágado Trachemys scripta elegans foi observado em todas as preparações o fígado ocupando grande parte da cavidade celomárica e dividindo-se em lobo direito e esquerdo, como também foram observados por Ashley (1969), Kassab et al. (2009), Silva et al. (2011) e Moura et al. (2012). Moura et al. (2017) relataram que o fígado ocupa praticamente toda a cavidade celomática. Em jacaré (Caiman crocodilus crocodilos) Alvarado-Rico et al. (2012) relataram que há dois lobos direito e esquerdo formando o fígado, unidos por um istmo, fato observado nos cágados (iiguras 1 e 2 ).

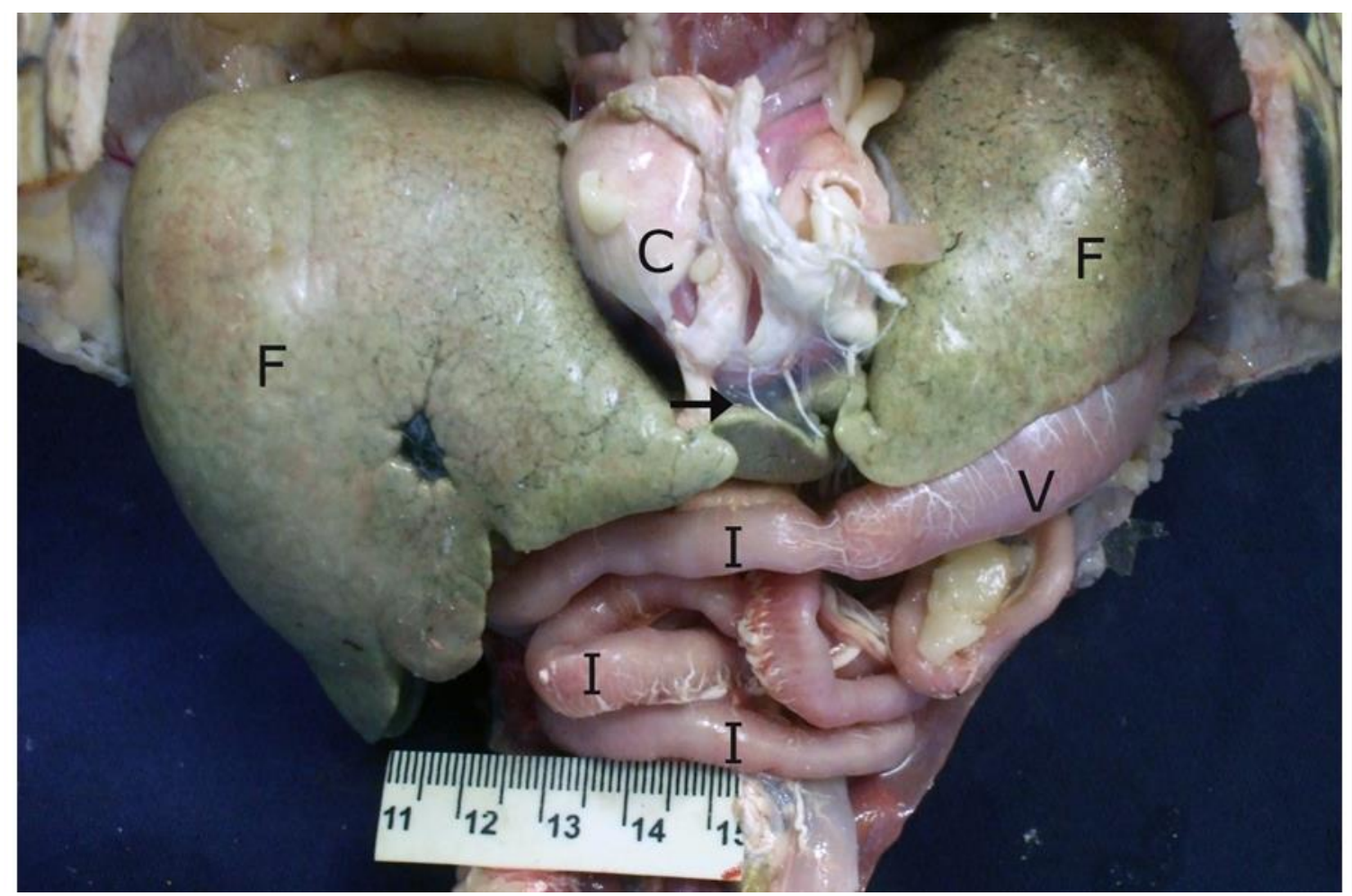

Figura 1. Fotomacrografia das vísceras de um Trachemys scripta elegans, onde se observa o fígado (F) ao redor do coração $(\mathbf{C})$. Nota-se também o ventrículo gástrico $(\mathbf{V})$ e intestinos (I). A seta indica os ligamentos coronarianos, que ligam o coração ao fígado.

Os lobos hepáticos apresentaram-se unidos por uma fina faixa de tecido, um istmo, onde se evidenciou inserido o coração, fato também confirmados por Girgis (1962), Ashley (1969), Yeager (1973), Romer \& Parson (1985), Bezuidenhout (1986), Wyneken (2001), Rossini (2003), Matsumoto (2004), Machado Júnior et al. (2007) e Alvarado-Rico et al. (2012). Moura et al. (2012) apresentaram o fígado de formato retangular, ao contrário do que foi evidenciado no presente trabalho os lobos hepáticos 
com massas irregulares em cada antimero. Pode-se supor que mesmo tratando-se de cágados há diferenças significativas entre ambos os lobos hepáticos em cada antímero (Figura 1).

No presente estudo, observou-se que o ventrículo gástrico do cágado Trachemys scripta elegans é circundado pelo lobo esquerdo do fígado, de acordo com os estudos de Machado Júnior et al. (2007) em muçuã e Ashley (1969).

A vesícula biliar do cágado Trachemys scripta elegans pode estar envolta pelos lobos hepáticos direito e esquerdo, ou exposta como demonstraram Moura et al. (2012) em P. geoffroannus. Essa observação sugere que é necessária para uma maior proteção da estrutura pelo parênquima hepático nessa espécie (Figuras 1 e $\underline{2}$ ).

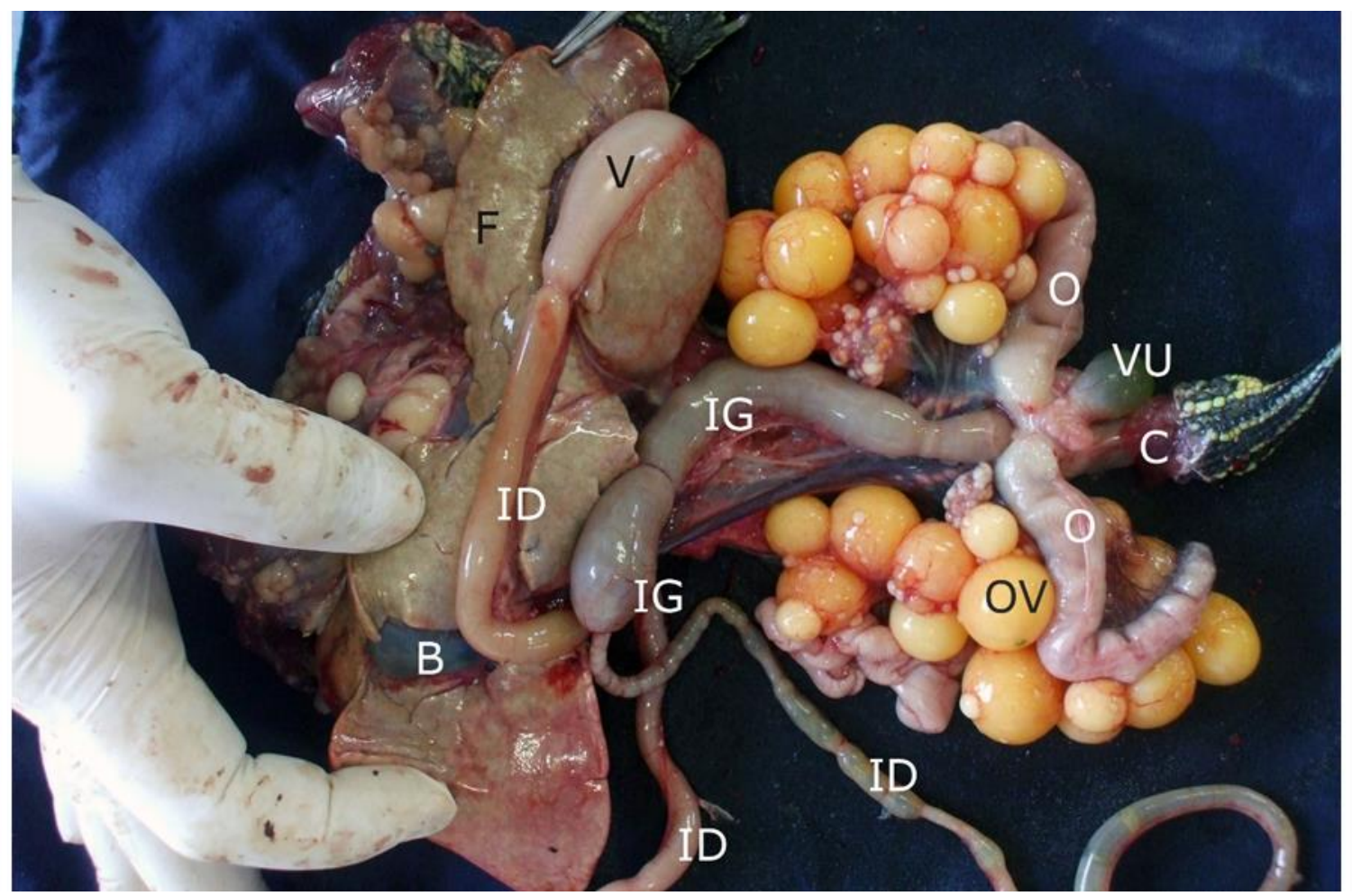

Figura 2. Fotomacrografia das vísceras de um Trachemys scripta elegans, onde se observa o fígado (F) com a vesícula biliar (B) envolvendo o ventrículo gástrico (V). O intestino delgado (ID) apresenta maior comprimento quanto comparado ao intestino grosso (IG). O intestino grosso abre-se na cloaca (C) passando dorsal a vesícula urinária (VU), que apresenta sintopia com o oviduto $(\mathbf{O})$ e ovário $(\mathbf{O V})$.

\section{Caracterização da veia porta-hepática}

O sistema porta-hepático foi descrito por Girgis (1962) nos cágados (Trionyx triunguis), Ashley (1962) em tartarugas, Yeager (1973) nos lagartos (genus Varanus), Romer \& Parsons (1985) e Storer et al. (1995) em répteis, Diaconescu (1985) nos cágados, Crawford \& Jackson (1989) nas tartarugas, Schaffner (1998) nos répteis. Alves Pinto et al. (1999) Cairina moschata o consideram como o sistema que transporta o sangue venoso do ventrículo gástrico, intestinos delgado e grosso, pâncreas e baço em direção ao fígado onde são realizadas várias funções.

Nas Trachemys scripta elegans a veia porta-hepática mostra dois locais de aprofundamento na superfície visceral do fígado, estando um à direita nas proximidades da vesícula biliar e outra à esquerda denominada veia porta-hepática transversa, de acordo com os resultados dos autores como Yeager (1973), Diaconescu (1985) e Wyneken (2001). Além disso, no sentido da esquerda para à direita, as veias gástricas, a veia pancreaticoduodenal cranial juntamente com o tronco das veias jejunais, além de um número variável das veias esplênicas e das veias mesentéricas cranial e caudal (Figura 3).

A veia porta-hepática aprofunda-se em dois locais na superfície visceral do fígado, um na porção direita próximo a vesícula biliar e outro, à esquerda, entre a veia porta-hepática transversa como 
observaram Nishida et al. (1969), Baumel (1986) e Santos et al. (2009), em aves, que são aparentados com os cágados estudados.

Em três animais (3/15) verificou-se que havia um único vaso que drenava o cólon transverso diretamente para o fígado, fato não observado por nenhum dos autores consultados. Nestes animais, a veia jejunal drenava diretamente para a veia porta-hepática, sem participar do tronco das veias jejunais.

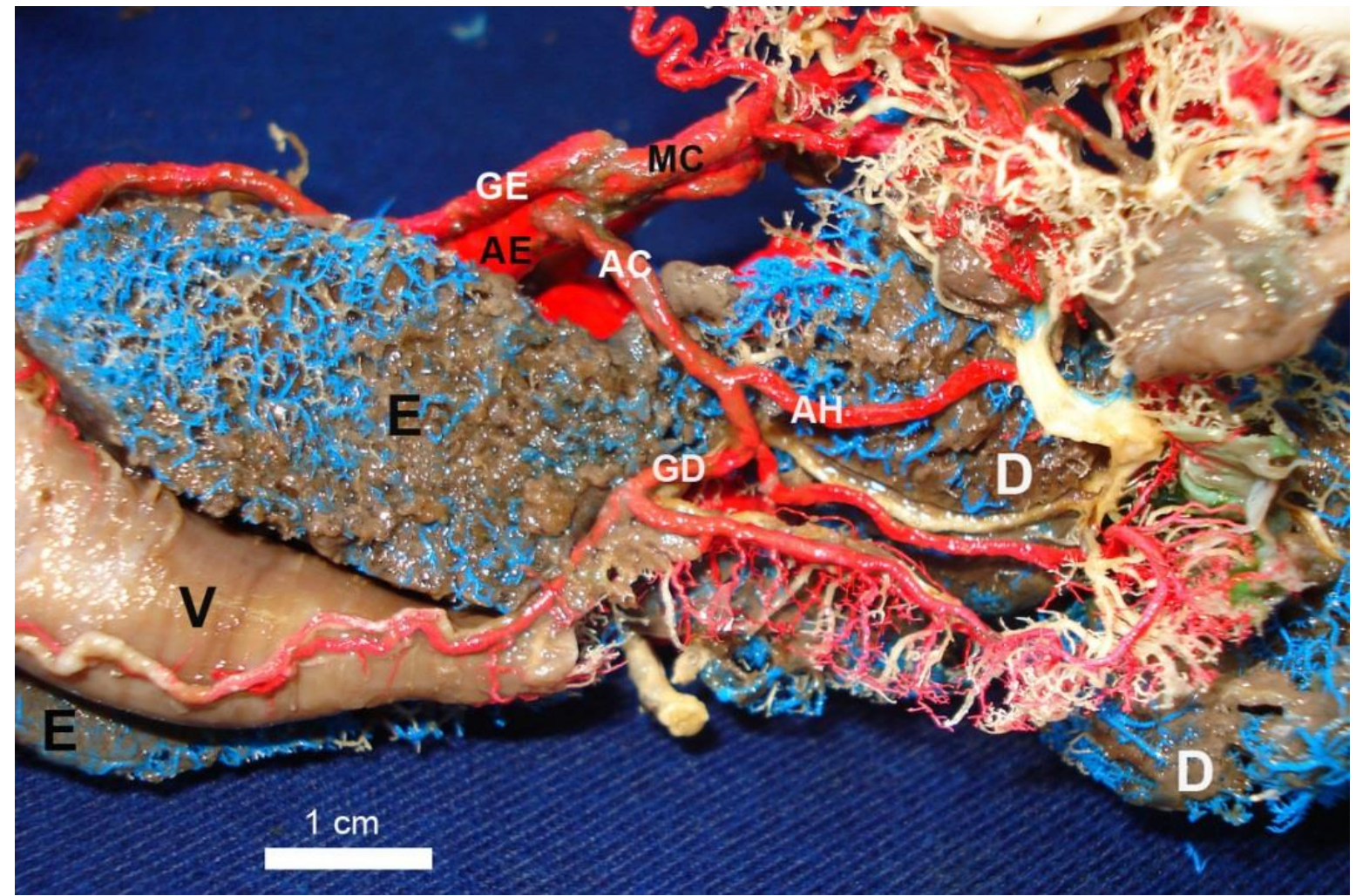

Figura 3. Fotografia das vísceras da cavidade pleuroperitoneal, semicorroída, onde as artérias foram injetadas com acetato de vinila na cor vermelha e as veias injetadas em azul e branco. Observa-se os lobos hepáticos direito (D) e esquerdo (E) drenados pelas veias hepáticas. Notar que a aorta esquerda (AE) emite três ramos: artéria gástrica comum esquerda (GE), artéria celíaca (AC) que emite as artéria gástrica comum direita (GD) e artéria hepática (AH), e artéria mesentérica cranial (MC).

O baço nestes animais avizinha-se com o pâncreas, apresentando de dois a três vasos que por sua vez desembocam na veia porta-hepática. Cranialmente ocorre anastomose com a veia mesentérica caudal em tronco comum com as veias jejunais, descrição também feita por Alves Pinto et al. (1999) em patos domésticos.

Alves Pinto et al. (1999) observaram, em patos doméstico, a presença de uma veia porta-hepática transversa recebendo as veias pancreáticoduodenais cranial e caudal e as veias gástricas, também descrita por Girgis (1962). A veia porta-hepática transversa também foi observado no material do presente trabalho (Figura 3). A presença da veia hemorroidária caudal, que drena a parte mais caudal do reto, unindo a veia mesentérica caudal e as veias jejunais, foi observado neste trabalho. Evidenciou-se nas Trachemys scripta elegans a veia mesentérica caudal penetrando no baço, e outros ramos no início do cólon uniram-se ao tronco das veias jejunais formando a veia porta-hepática.

\section{Conclusões}

Pode-se concluir com o presente estudo que o fígado, no cágado Trachemys scripta elegans, é volumoso e ocupa grande parte da cavidade celomática apresentando dois lobos distintos separados pelo coração, e a este unido pelo ligamento coronariano. A veia porta-hepática juntamente com sua auxiliar, a veia porta-hepática transversa, drenam o sangue do ventrículo gástrico, intestinos, baço e pâncreas em direção ao fígado. 


\section{Agradecimentos}

Fundação de Amparo à Pesquisa do Estado de São Paulo (FAPESP) pelo apoio financeiro (Processo $\left.n^{\circ} .2007 / 59452-7\right)$.

\section{Referências bibliográficas}

Alvarado-Rico, S., García, G., Céspedes, R., Casañas, M., \& Rodríguez, A. (2012). Caracterización morfológica e histoquímica del hígado de la baba (Caiman Crocodilus Crocodilus). Revista de la Facultad de Ciencias Veterinarias, 53(1):13-19.

Alves Pinto, M. R. A., Ribeiro, A. A. C. M., de Souza, W. M., Miglino, M. A., \& Machado, M. R. F. (1999). Estudo do sistema portal hepático no pato doméstico (Cairina moshata). Brazilian Journal of Veterinary Research and Animal Science, 36(4), 173-177.

Ashley, L. M. (1969). Laboratory anatomy of the turtle. WC Brown.

Baumel, J. J. (1993). Handbook of avian anatomy: nomina anatomica avium. In Publications of the Nuttall Ornithological Club (USA). Publications of the Nuttall Ornithological Club (USA).

Bezuidenhout, A. J. (1986). The topography of the thoraco-abdominal viscera in the ostrich (Struthio camelus). Onderstepoort Journal of veterinary Research, 53(11):11-117.

Crawford, G. \& Jackson, J.R. (1988) Cardiovascular system. Perspective and Research, Cidade: editora, 1989.

Cubas, Z. S., Silva, J. C. R., \& Dias, J. L. C. (2014). Tratado de animais selvagens-medicina veterinária. Editora Roca.

Diaconescu, N. (1985). Contributions to the evolutionary study of the liver afferent veins. Morphologie et embryologie, 31(1): 17-23.

Girgis, S. (1962). Anatomical and functional adaptations i-n the venous system of a diving reptile, Trionyx triunquis (FOR. SKAL). In Proceedings of the Zoological Society of London, 138 (3): 355377. Oxford, UK: Blackwell Publishing Ltd.

Hildebrand, M., \& Goslow, G. (2006). Análise da estrutura dos vertebrados. $2^{a}$ edição. Atheneu Editora.

Isaza, R., Ackerman, N., \& Jacobson, E. R. (1993). Ultrasound imaging of the coelomic structures in the Boa constrictor (Boa constrictor). Veterinary Radiology \& Ultrasound, 34(6), 445-450.

Kassab, A., Shousha, S., \& Fargani, A. (2009). Morphology of blood cells, liver and spleen of the desert tortoise (Testudo graeca). The open anatomy Journal, 1: 1-5.

Machado Júnior, A. A., Sousa, A. L., Carvalho, M. A. M., Santos, F. C. F., \& Alves, F. R. (2005). Anatomia do fígado e vias bilíferas do muçuã (Kinosternon scorpioides). Archives of Veterinary Science, 10(2), 125-133.

Matsumoto, F.S. Topografia e Morfologia das Vísceras do Periquito Australiano (Melopsittacus undulatus). 2004. Monografia, Centro Universitário da Fundação de Ensino Octávio Bastos.

Moura, L R, Santos, A. L. Q., Belleti, M. E., Vieira, L. G., Orpinelli, S. R. T., \& Simone, S. B. S. (2017). Morphological aspects of the liver of the freshwater turtle Phrynops geoffroanus Schweigger, 1812 (Testudines, Chelidae). Journal of Morphological Sciences, 26(3-4), 129-134.

Moura, Léa Resende, Santos, A., Beletti, M. E., Vieira, L. G., Orpinelli, S. R., \& Alves Júnior, J. R. (2012). Morphological aspects of the liver of the Podocnemis expansa (Testudines, Podocnemididae). Journal of Morphological Sciences, 26(3-4), 159-166.

Nishida, T., Paik, Y. K., \& Yasuda, M. (1969). Comparative and topographical anatomy of the fowl. 58. Blood vascular supply of the glandular stomach (ventriculus glandularis) and the muscular stomach (ventriculus muscularis). Nihon juigaku zasshi. The Japanese journal of veterinary science, 31(2): $52-70$.

Pough, F. H., Heiser, J. B., \& McFarland, W. N. (2003). A vida dos vertebrados (Vol. 3). Atheneu Editora.

Romer, A. \& Parson, T. (1985). Anatomia comparada dos vertebrados. São Paulo: Atheneu, 388-390. 
Rossini, M. (2012). Características macroscópicas, histológicas e histoquímicas del sistema digestivo de la baba (Caiman crocodilus crocodilus). Revista de la Facultad de Ciencias Veterinarias, 1:1319.

Rossini, T. C., Borba, C. C., Menconi, A., Maia, M. O., Bombonatto, P. P., \& Pereira, C. C. (2009). Veias do sistema porta-hepático em gansos domésticos. Pesquisa Veterinária Brasileira, 327-332.

Santos, T. C., Borba, C. C., Menconi, A., Maia, M. O., Bombonatto, P. P., \& Pereira, C. C. (2009). Veias do sistema porta-hepático em gansos domésticos. Pesquisa Veterinária Brasileira, 327-332.

Schaffner, F. The Liver In: Gans, C. (Ed.). (1998). Biology of the Reptilia: Morphology G: visceral organs (Vol. 19). Academic Press.p.485-488.

Silva, G. F. N., Freire, V. T. O., Matos, W. C. G., Pereira-Neto, J., Seyfert, C. E., Andrade, N. S., \& Faria, M. D. (2011). Dimensions, mass and volume of the liver of turtles (Trachemys scripta elegans Wied, 1839). Journal of Morphological Science, 28, 235-239.

Storer T. I., Usinger R. L., Stebbins R. C., Nybakken J. W. (1995) Zoologia geral. 6th ed. Nacional, São Paulo, p. 837.

Tranquilli, W. J., Thurmon, J. C., \& Grimm, K. A. (2013). Lumb and Jones' veterinary anesthesia and analgesia. John Wiley \& Sons.

Wyneken J. (2001) The Anatomy of Sea Turtles. U.S. Department of Commerce NOAA Technical Memorandum NMFS-SEFSC-470, 172p.

Yeager V. L. (1973). The vascular anatomy of the liver of the monitor lizard (genus Varanus). American Journal of Anatomy, 136(4), 441-453.

Zug, G. R., Vitt, L. J., \& Caldwell, J. P. (2001). Herpetology: an introductory biology of amphibians and reptiles. Elsevier.

Recebido: 16 de outubro, 2019.

Aprovado: 22 de novembro, 2019.

Publicado: 25 de março, 2020.

Licenciamento: Este artigo é publicado na modalidade Acesso Aberto sob a licença Creative Commons Atribuição 4.0 (CC-BY 4.0), a qual permite uso irrestrito, distribuição, reprodução em qualquer meio, desde que o autor e a fonte sejam devidamente creditados. 